\title{
LA DÉMOCRATIE, L'INTOLÉRANCE POLITIQUE ET LES DROITS HUMAINS : LE BRÉSIL ET LA CRISE ACTUELLE
}

DEMOCRACY, POLITICAL INTOLERANCE AND HUMAN RIGHTS: BRAZIL AND THE CURRENT CRISIS

Eduardo C. B. Bittar

University of São Paulo. Faculty of Law, Brazil.

ecbbittar@gmail.com

Reçu: 2016-05-14. Accepté: 2016-09-19.

Résumé: L'atmosphère d'intolérance dans les démocraties contemporaines est incompatible avec le concept de démocratie, dans un sens plus large ; ce qui présente clairement la corrélation étroite entre la démocratie et les droits humains, et aussi comme la négation des droits humains sont révélés dans les périodes de crise économique et politique. Cet article essaie de traiter de la question de la démocratie dans la vie quotidienne, en abordant des questions de politique actuelle dans la réalité brésilienne contemporaine.

Mots-Clefs: Intolérance politique - Crise Politique - Droits Humains

Abstract: The atmosphere of intolerance in contemporary democracies is incompatible with the concept of democracy in a wider sense, which shows the close correlation between democracy and human rights, and also how human rights deficits are exposed in full force in periods of strong economic and political crisis. This article seeks to address these issues so intersected in contemporary Brazilian reality, addressing the issue of the daily derepression.

Keywords: Political Intolerance - Political Crisis - Human Rights

\section{L'INTOLÉRANCE POLITIQUE ETLES DROITS HUMAINS}

Le Brésil a une 'image internationale’ qui est étroitement associée 
à la 'fête', à la 'samba' et à la 'cordialité' du peuple. ${ }^{1}$ Celàfonctionne aussi bien quand il s'agit de prendre ces traits de la culture brésilienne comme 'clé' de autocompréhension, ou de parler de l'image de soi'que nous avons internement dans notre pays.Mais, cette idéologie trompe l'analyse, quand on veut regarder les rélations de la vie quotidienne dansla réalité concrète. Soità cause de la sociologie romantique, soit à cause du marketing mondial, soit à cause du métissage ethno-racial historique, nous avons cette vision aveugle comme manifestation d 'une idéologie qui nous empêche de confronter nos plus concrets problèmes du quotidien.

Dans le contexte de l'actuelle crise économique et politique, ces problèmes émergent des profondeurs des relations sociales, et se revèlent, en démontrant que lemasque de notre 'civilité brésilienne'a disparu sous nos yeux. Ainsi, il est vrai de dire que, dans le Brésil d'aujourd'hui, la vie quotidienne est plus marquée par la distension des relations personnelles, et que nous vivons au 'bord du rasoir' ('fio da navalha'), dans un scénario de polarisation politique, d'extrêmesde l'opinion publique, d'instabilité institutionnelle et de paralysie économique.

Certes, d'un point de vue historique, la crise économique a toujours réveillé les 'fantômes' les plus terribles qui existent, surtoutsi l'on prend en considération la peur généralisée du peuple et les sentiments manipulés par les media et le gouvernement, favorisant ainsi une atmosphère d'intolérance politique et sociale.

Mais, la crise économique n'est pas locale et n'est pas non plus un privilège brésiliendu moment.La crise est mondiale, et facilite l'extrémisme dans toutes les parties du monde, comme ont montré récemment les actes de terrorisme du 13 Novembre 2015, dans la ville de Paris.

Toute période de crise présente une opportunité pour la manifestation descontradictions jusqu'alors cachées. Ce qui était en suspens, maintenant apparaît comme une fatalité. Chez nous, au Brésil, la contradiction est la consolidation de la démocratie en suspens; en France, la tension dévint dela situation non résolue de l'immigration. $C^{\prime}$ estdans de tels moments que la crise fait 'la civilisation' se soumettre aux épreuves plus difficiles, en faisant réagir la culture et en faisant se manifester 'la barbarie quotidienne' qui est camouflée sous la protection du mot 'civilisation', et plus spécialement du mot 'civilisation moderne'. ${ }^{2}$

\footnotetext{
1 "Often described as a difficulty to separate personal and impersonal attitudes in the public space, associated with a spirit of cordiality (Holanda, 1936/1997) or expressed in the difficulty to deny favor to friends (Damatta, 1991, 1999) and in the ability to always find a way to help a friend (the famous brazilianjeitinho), such orientation contrasts with the rules that predominate in the market and in State bureaucracy" (Cardoso de Oliveira, Luís Roberto, Equality, dignity and fairness: Brazilian citizenship in comparative perspective, in Critique of Anthropology, 33(2), 131-145, 2013, p. 132).

2 Cf. Adorno, Theodor, Horkherimer, Max,Dialética do esclarecimento: fragmentos filosóficos,
} 
Leprocessus de modernisation implique des avances et des reculs, sélonles observations de la Dialectique des Lumières (1947), de Theodor Adorno et Max Horkheimer. ${ }^{3}$ Celui ciest un important avertissement historique signalé par la Frankfurt Schüle, qui analyse les pathologies sociales dans la modernité, pour faire resortir la critique des éléments opprimant la liberté. Cettre analyse nous fait voire que 1' 'histoire de la modernité'n'est pas seulement l' histoire de la liberté'. Les jeux sociaux impliquant des étapes de l'émancipation ne sont pas libres de tensions, de conflits et de contradictions. Ainsi, la modernité nous fait tout le temps des surprises;comme par exemple, quand la liberté acquise est immédiatement minée par une innovation de la technologie, par des scénarios de crise économique, par un nouvel accord des forces politiques, ou par un scénario d'incertitudes sociohistoriques, ou même, par la force de la terreur globale.

Nous traversons une période difficile, de fortes tempêtes politiques, de scandales de corruption, de perte de légitimité du gouvernement, mais toute difficulté ne peut pas nous rendreaveugle à poin de ne pas voire clairement que l' intolérance politique du passé a fait trop de victimes dans l'histoire récente, et qu'il est bien temps de se tourner vers les promesses des Lumières. ${ }^{4}$ Par conséquent, la préservation des droits humains ne peut pas subir de revers, soitau nom du 'conservatisme politique', dans le cas du Brésil, soit au nom de la 'sécurité nationale', dans le cas de la France. La menace de retour en arrière dans la culture du respect des droits humains, dans la lutte pour la consolidation de la démocratie et dans la croyance aux institutions de protection de la légalité ne peut pas nous faire revenir en arrière et retourner à un passé récent où la politique cachait le totalitarisme et l'autoritarisme.

Au Brésil, la situation est plus délicate encore, étant donné que la faiblesse de notre démocratie. Les résultats d'avaliation du Democracy Index indique que le Brésil est la 51ème démocratie du monde, dans une rélation de 167 pays analysées, et qu'il a perdu des positions (il était la 47ème position) à cause de l'actuelle crise politique. En faite, après la dictature civil-militaire de 1964-1985, et déjà passé la période de la restauration démocratique depuis l'édition de la Constitution Fédérale de 1988, il ya eu deux demandes de impeachment (destitution constitutionelle) présentées contre des Présidents élus par le vote populaire. Dans le premier cas, la destitution du Président Fernando Collor, après des manifestations populaires desjeunes 'caras pintadas'; dans le second cas, l'impeachment de la Présidente Dilma Roussef, encore en discussion,

São Paulo, 1985, ps. 12 à 27.

3 Cf. Adorno, Theodor, Horkheimer, Max,Dialética do esclarecimento: fragmentos filosóficos, São Paulo, 1985, p. 19.

4 Garapon, Antoine, O guardador de promessas: justiça e democracia, Lisboa, 1998, ps. 36-37. 
après les manifestations populaires (pour et contre).

Mais, du point de vue historique pour la consolidation de la démocratie brésilienne, le plus tragique est la constatationdans la réalité contemporaine de certains types de marques du quotidien: a.) hate speech (discours de haine) en large propagation virale, dans les sites et partout sur l'internet (Facebook ; Instagram); ${ }^{5}$.) la violence pandémique; ${ }^{6} \mathrm{c}$.) les inégalités socio-économiques; d.) des expressions de l'opinion publique autoritairedemandant le retour de la dictature; e.) les tentatives législatives pour supprimer certains droits humains, en particulier dans le domaine des droits des enfants et des adolescents; f.) le processus pas finit de justice de transition; ${ }^{7}$ g.) la dégradation du dialogue politique dans la sphère publique; $h$.) la forte pression des medias sur le gouvernement.

\section{DéMOCRATIE DaNS La VIE QUOTIDIENNE}

La démocratie brésilienne vitles mêmes questions qui affectent une grande partie des démocraties contemporaines autour du monde, parce qu'on vit dans un scénario d'extrêmes. C'est pour ça qu'on a besoin maintenant de stabiliser la démocratie et non de la revoquer; on a besoin de l'approfondir et de la rendre présente où elle ne l'était pas. C'est pour cela que la conception de démocratie dont on a besoin, aujourd'hui, n'est pas celle de la traditionelle 'démocratie politique'. On a besoin d'une conception de démocratie plus large.

Cela est très présent, parce que la démocratie brésilienne contemporaine ade fortes batailles à vaincre au niveau de la culture et au niveau de l'économie, au niveau de la justicesociale et au niveau de la citoyenneté, au niveau de l'éducation et auniveau de l'opinion publique, aussi importantes que les batailles au niveau politique. Je comprends ça comme d'importantes étapes de préparation pour surmonter le cycle de prémodernité qui empêche le Brésil de vaincre les inégalités économiques, les relations sociales violentes, et l'autoritarisme social.

$C^{\prime}$ est à cause de ça que peut commencer à sortir dans le débat contemporain autour du terme 'démocratie', le point de vue selon lequel la réalisation et la radicalisation de la démocratie signifient, aujourd'hui, plus que la réforme du'système politique', entraînant la notion de démocratie de la vie quotidienne, une sorte de démocratie

5 Rothenburg, Walter Claudius; Stroppa, Tatiana, Liberdade de expressão e discurso de ódio: o conflito do discurso nas redes sociais, in Anais do III Congresso Internacional de Direito e contemporaneidade: mídias e direitos da sociedade em rede, http:/www.ufsm.br/ congressodireito/anais, Acesso em 30.12.2015.

6 Cf. Pinheiro, Paulo Sérgio; Almeida, Guilherme A., Violência urbana, 2003, p. 5 a 24.

7 Cf. MINISTÉRIO DA JUSTIÇA. Relatório final. Brasília: MJ, Comissão Nacional da Verdade, In: http://www.cnv.gov.br. 
pour les rélations quotidiennes entre citoyens, toujours pratiquéesur la base de la négociation rationnelle, du dialogue et du renforcement des valeurs des droits humains. Cela signifie un profond processus de dérépression du quotidien des relations humaines.

Cela implique la conscience des citoyens de ce qui est commun et de ce qui fait chacun faire partie d'une communauté d'intérêts. La démocratie n'est pas cachée dans une boîte, qu'on appelle 'institutions politiques', le Congrès, le Tribunal, la Maîrie; ${ }^{8}$ elle est effectivement présente aux liens sociaux capables de construire spontanément des pratiques démocratiques où des valeurs sociales de convivialité. Ceci un point très important de cet exposé, parce qu'il touche directement la question de la formation d'une culture sociale démocratique. Cela demande une attitude bien radicale devant la vie, soitcollective, soitindividuelle, et la capacité d'intérioriser le nous dans le moi, et le moi dans le nous, et de faire du dialogue une méthodede vie desvaleurs républicaines.

Qui mieux es connaissant l'histoire et les injustices du passé, et sachant que notre passé nous pèse énormément dans le présent (esclavage, génocide des indiens, violences du quotidien, déficits des politiques publiques, république inachevée), on sait que le plus important c'est de chercher, dans l'horizon d'un futur proche, les moyens de la citoyenneté et de la consolidation de la démocratie comme les seules alternatives politiques possibles aux problèmes de notre réalité. La préservation de la démocratie et le progrès de la démocratie ne peuvent pas ignorer ces éléments.

Par conséquent, lors de la formulation de la notion contemporaine de 'démocratie', il faut tenir en compte ces aspects abordés ici plus profondément. Il faut aussi examiner comment la radicalisation de la démocratie pourra se faire sans une culture de démocratie dans la vie sociale. Si l'on $\mathrm{n}$ 'est pas pas capable d'actualiser les pratiques en harmonie avec l'évolution des ces nouvelles frontières du concept, la 'démocratie politique' sera toujours passible d' 'interruptions', $\mathrm{d}^{\prime}$ 'indéfinitions' et d'autres 'instabilités'. Ces résultats d'analyse définissent les directionsdu futur, et indiquent par eux-mêmes, les conclusions. Il faut stabiliser la démocratie dans la vie, au sens d'une pratique quotidienne, exactement où le moi se confond avec le tu, et où les valeurs des droits humains sont les plus gravement maltraitées.

\section{Conclusions}

L'atmosphère d'intolérance politique au Brésil contemporainexposelaréelcapacité de ladémocraties'affirmer, se consolider et de réaliserlaculturedesdroitshumains, si nécessaire

8 Garapon, O guardador de promessas: justiça e democracia, 1998, ps. 43-44. 
commegarantie de respectdanslesexchangessociaux.

La crise politique est sûrementlaconséquence de la crise économique, mais lesinstitutions de justice suiventenpleineactivité, cequipermet d'affirmer que lecontroldémocratique est déjà une manière de forcerlesystèmejuridique à s'imposersurlesystème politique, cequis'affirmecommedémonstration d'autonomie et de puissancequicommence à créer une nouvelle histoire de combat à lacorruption et de division entre leprivée et le publique.

La crise expose le problème de la proximité du gouvernement des entreprises privées, et les scandales montrent la nécessité de reforme politique et de punition aux auteurs des actes criminels. Mais, dans ce temps-là, des démonstration de participation de la population est très bien réçu comme mouvement pour ou contre la figure de la Présidente, mais surtout, contre chaque acte de corruption où de atentat contre l'État démocratique. L'activité citoyenne a été très mobilisé, spécialment après les années 2013-2016, ce qui démontrent que la poursuite d'une nouvelle culture politique sera bien venu aux prochaines années.

Mais, si les manifestation populaires sont très positives, la violence et l'intolérance sont des choses à régreter. $C^{\prime}$ est dans ce point-là qui on va voire les ménaces aux rélations entre les gens, entre les familiers, entre les amis, ce qui fait soner l'alarme de la culture des droits humains. Par conséquent, la question de la démocratie au quotidien et des valeurs républicaines pour la coexistence pacifique sont aussi décisives pour l'affirmation active de la culture de la dignité de tous, pour la réalisation des droits humains et pour le respect à la loi. Tout ceci di assez combien la fragilité de la démocratie va de front avec l'irrespect des valeurs sociales et quotidiennes les plus élémentaires des droits humains.

\section{REFERENCES}

ADORNO, Theodor W.; HORKHEIMER, Max. Dialética do esclarecimento: fragmentos filosóficos. Tradução de Guido Antonio de Almeida. Rio de Janeiro: Jorge Zahar, 1985.

BAUMAN, Zygmunt. O mal-estar da pós-modernidade. Tradução de Mauro Gama; Cláudia Martinelli Gama. Rio de Janeiro: Jorge Zahar, 1998.

BECK, Ulrich (org.). Hijos de lalibertad. Traducción de Mariana RojasBermúdez. México: Fondo de Cultura Económica, 2006.

BENOIST, Alain de. The currentcrisisofdemocracy, Telos, n. 156, p. 7-23, New York, Télos Press, 2011.

BERMAN, Russell A. Humanities and the public sphere, Telos, n. 159, p. 171-186, New York, Telos Press, 2012. 
BITTAR, Eduardo C. B. O direito na pós-modernidade.2.ed. Rio de Janeiro: Forense Universitária, 2009.

. Democracia, justiça e emancipação social: reflexões jusfilosóficas a partir do pensamento de Jürgen Habermas. São Paulo: Quartier Latin, 2013.

BOHMAN, James. The coming of age of deliberative democracy, in The Journal of Political Philosophy, volume 6, Number 4, Blackwell Publishers, Oxford/ Malden, 1998, pp. 400-425.

BRONNER, Stephen Eric. Reclaiming the Enlightenment: toward a politics of radical engagement. New York: Columbia, 2004.

CARDOSO DE OLIVEIRA, Luís Roberto, Equality, dignity and fairness: Brazilian citizenship in comparative perspective, in Critique of Anthropology, 33(2), 131-145, 2013.

COHEN, Joshua, Democracy and liberty, in Deliberative democracy (Elster, John, org.), New York, Cambridge University Press, 1998, ps. 185-231.

CRETTIEZ, Xavier. As formas da violência. Tradução de Lara Christina de Malimpensa; Mariana Paolozzi Sérvulo da Cunha. São Paulo: Loyola, 2011.

DAGNINO, Evelina, Sociedad civil, espacios públicos y construción democrática em Brasil: límites y posibilidades, in Sociedad civil, esfera pública y democratización em América Latina: Brasil (Dagnino, Evelina, coord.), UNICAMP, Fondo de Cultura Económica, México, p. 369-395, 2002.

DAHL, Robert A. A democracia e seus críticos. Tradução de Patrícia de Freitas Ribeiro. São Paulo: Martins Fontes, 2012.

DAMATTA, Roberto. Carnavais, malandros e heróis: para uma sociologia do dilema brasileiro. 6.ed. Rio de Janeiro: Rocco, 1997.

FISHKIN, James S.; LASLETT, Peter. Debating Deliberative Democracy. Oxford: Blackwell Publishing Limited, 2003.

FORST, Rainer. Contextos da justiça: filosofia política para além de liberalismo e comunitarismo. Tradução de Denilson Luís Werle. São Paulo: Boitempo, 2010.

GARAPON, Antoine. O guardador de promessas: justiça e democracia. Tradução de Francisco Aragão. Lisboa: Instituto Piaget, 1998.

. Bem julgar: ensaio sobre o ritual judiciário. Tradução de Pedro Felipe Henrique. Lisboa: Instituto Piaget, 1999.

GOYARD-FABRE, Simone. O que é democracia? Tradução de Claudia 
Berliner. São Paulo.

HÄBERLE, Peter, Pluralismo y Constitución: estudios de Teoría Constitucional de la sociedad abierta. Traducción de Emilio Mikunda. Madrid: Tecnos, 2002.

HABERMAS, Jürgen. Faktizität und Geltung: Beiträge zur Diskurstheorie des Rechts und des demokratischen Rechsstaats. Frankfurt am Main: Suhrkamp, 1998.

HOBSBAWN, Eric. Globalização, democracia e terrorismo. Tradução de José Viegas. São Paulo: Companhia das Letras, 2007.

. Era dos extremos: o breve século XX. Tradução de Marcos Santarrita. 2.ed. São Paulo: Companhia das Letras, 2002.

HOLANDA, Sérgio Buarque de. Raízes do Brasil. 26. ed. São Paulo: Companhia das Letras, 2004.

BRASIL. MINISTÉRIO DA JUSTIÇA. Relatório final. Brasília: MJ, Comissão Nacional da Verdade, In: http://www.cnv.gov.br. Acesso em 30.12.2015.

PINHEIRO, Paulo Sérgio; Guilherme A. Almeida. Violência urbana. São Paulo: Publifolha, 2003.

RIBEIRO, Darcy. O povo brasileiro: a formação e o sentido do Brasil. São Paulo: Companhia das Letras, 1995.

ROTHENBURG, Walter Claudius; STROPPA, Tatiana, Liberdade de expressão e discurso de ódio: o conflito do discurso nas redes sociais, in Anais do III Congresso Internacional de Direito e contemporaneidade: mídias e direitos da sociedade em rede, In http://www.ufsm.br/ congressodireito/anais, Acesso em 30.12.2015.

VELHO, Gilberto. Individualismo e cultura: nota para uma antropologia da sociedade contemporânea. 7.ed. Rio de Janeiro: Jorge Zahar, 2004. 